Aus dem Forschungsinstitut fur die Biologie landwirtschaflicher Nutztiere (FBN), Dummerdstorf

DELIA MICKLICH und HEIDE-DÖRTE MATTHES

\title{
Die Weidehaltung von Sauen verschiedener Rassen auf FluBauenstandorten
}

\begin{abstract}
Summary
Title of the paper: Outdoor keeping of sows of different breeds in a location of a floodplain wood The present investigations show results of four different breeds of pigs (Schwerfurter Meatrace, German SattleBack, Duroc and Mangalica) in the nationalpark "Brandenburgische Elbtalaue“. The aim of the investigations are checking the pigs with regard to their rooting activity, behaviour and their litter.
\end{abstract}

Key words: sows, outdoor keeping, rooting activity, behaviour, litter

\section{Zusammenfassung}

Es werden Ergebnisse einer Freilandhaltung von vier verschiedenen Schweinerassen (Schwerfurter, Deutsches Sattelschwein, Duroc und Mangalitza) auf Flußauengrünland in der „Brandenburgischen Elbtalaue“ dargestellt. Ziel war die Prüfung von vier in ihren Eigenschaften unterschiedlichen Schweinerassen hinsichtlich ihrer Wühlaktivität, ihres Verhaltens sowie ihrer Wurfleistungen.

Schlüsselwörter: Sauen, Freilandhaltung, Wühlaktivität, Verhalten, Wurfleistung

\section{Einleitung}

Die Schweinehaltung in diesem Jahrhundert ist überwiegend durch die Stallhaltung gekennzeichnet. Zwar gab es nach dem 2. Weltkrieg verstärkt Weidehaltung bis zur Freilufthaltung von Schweinen (HOFMANN, 1952; SCHLEGEL und RITTER, 1959), jedoch traten diese Haltungsformen mit zunehmenden Leistungsforderungen an die Tiere immer mehr zurück. Arbeitssparende Aufstallungsformen, Züchtung und ausgewogene Fütterung führten zu hohen Mast- und Schlachtergebnissen. Verlustgeschehen, Erkrankungen und ethische Überlegungen bewirkten eine Rückbesinnung auf artgerechtere Haltungsformen (SCHMIDT u.a., 1995). Sie führten letztlich zu Rahmenvorschriften im Tierschutzgesetz in Verbindung mit der Schweinehaltungsverordnung, die neben Regelungen der Haltung einzelner Nutzungsgruppen bei Sauen unter anderem Zeiten freier Bewegung vorgeben (GLODEK, 1992).

In Großbritannien führte ROADNIGHT im Jahre 1952 die ganzjährige Freilandhaltung nicht nur von güsten und tragenden, sondern auch von ferkelführenden Sauen wieder ein (MAJOR, 1986). Er verwendete als Muttertiere Schweine der Rasse Sattleback, die sich durch sehr gute Muttereigenschaften auszeichneten.

Zunehmend bedeutsam wird gegenwärtig in Deutschland die Freilandhaltung von Sauen hinsichtlich der ,artgerechten Tierhaltung“. Intensive Haltungsformen ent- 
sprechen zwar meist den hygienischen und arbeitswirtschaftlichen Anforderungen, sind aber hinsichtlich auftretender Verhaltensstörungen oft nicht so positiv zu beurteilen (SAMBRAUS, 1986).

Verursacht durch die besonderen Umweltbedingungen bei der Freilandhaltung hinsichtlich Klima und extensiver Haltung, werden von Tieren, die hierfür vorgesehen sind, folgende Eigenschaften gefordert (DURST und WILLEKE, 1994):

- Robustheit und Anspruchslosigkeit,

- Anpassungsfähigkeit an neue Situationen,

- guter Charakter, Ruhe, Friedfertigkeit,

- keine Geburtsschwierigkeiten,

- gute Muttereigenschaften,

- Gesundheit

- gute körperliche Verfassung und möglichst Pigmentierung.

Nachdem das Deutsche Weideschwein ausgestorben ist, wurden für die Weidehaltung in Deutschland überwiegend Landrassen genutzt. In England und Dänemark haben sich Kreuzungssauen mit 50\%igem Duroc-Anteil bewährt. Noch heute gilt das Mangalitza-Wollschwein in der Hutewirtschaft als robust und ist für das ganzjährige Leben im Freiland gut geeignet (GRÜNENFELDER, 1994). Diese Bewirtschaftungsform der Hutweiden ist in fast allen europäischen Ländern heute ausgestorben. Sie ist aber noch in den Save-Auen Kroatiens anzutreffen.

Die Intensivierung der Feldwirtschaft bei gleichzeitiger Flächenstillegung weniger guter Standorte erfordert neue Nutzungskonzepte. Eine Möglichkeit stellt die Freilandhaltung von Schweinen auf solchen Flächen dar. Beobachtungen zeigen, daß sich bei Einstellung der landwirtschaftlichen Nutzung auf nährstoffreichen Standorten in der Regel eine Hochstaudenflora entwickelt, die für längere Zeit den Gehölzanflug unterdrückt (MEISEL, 1973; REICHEL, 1977). Im Gegensatz zu brachliegenden Ackerflächen verhalten sich geschlossene Grasnarben relativ resistent gegenüber dem Aufkommen von Gehölzen (MEISEL, 1973; MEISEL und HÜBSCHMANN, 1973; REICHEL, 1977; RUNGE, 1984).

In vorliegend beschriebener Untersuchung werden Ergebnisse einer Freilandhaltung von vier verschiedenen Schweinerassen auf Flußauengrünland eines Versuches in der Brandenburgischen Elbtalaue dargestellt. Dieses Flußauengrünland wurde während der Sommermonate extensiv mit Schweinen beweidet, um das Weidefutter zu verwerten sowie den Verlauf der Sukzession auf den von Schweinen beweideten Flächen zu dokumentieren (MICKLICH, 1997). Ziel war nicht eine intensive Nutzung der Schweinebestände, sondern die Prüfung von vier in ihren Eigenschaften unterschiedlichen Schweinerassen hinsichtlich ihrer Wühlaktivität, ihres Verhaltens sowie einiger ermittelter Leistungen während der Freilandhaltung.

2.

Material und Methode

Die Sauen sind während der Vegetationsperiode 1994 (Juni bis November) ganztägig auf der Weide gehalten worden, wobei sie dort Schutzhütten aufsuchen konnten. Die Sauen im 2. bzw. 3. Wurf, bei annähernd gleicher Altersstruktur, hielt man rassen- 
getrennt mit jeweils einem Eber je Gruppe mittels eines doppelten Elektrozaunes, welcher in Höhen von $40 \mathrm{~cm}$ und $60 \mathrm{~cm}$ über dem Erdboden angebracht war. Die Flächen wurden mit 5 Schweinen / ha beweidet. Die Sauen ferkelten meist in den dafür zur Verfügung stehenden Abferkelhütten auf der Weide ab. Die nach 39-78 Tagen (Tab. 5) abgesetzten Ferkel blieben nicht auf den Flächen.

Im Freiland erhielten die Tiere eine tägliche Getreide-Kraftfuttergabe (Einzelfreßstände) von $1 \mathrm{~kg}$ (13 MJ ME/kg, 17\% RP) neben einer ad libitum Grasaufnahme. Die Ermittlung der gewichteten Futterwerte des Grünlandes (KLAPP und OPITZ VON BOBERFELD, 1988) ergab einen Wert von 5,2, was eine mittlere Einstufung bedeutet. Die Zufütterung in Einzelfreßständen ermöglichte eine gezielte Kraftfuttergabe der Tiere. Wasser erhielten die Tiere ständig ad libitum. Für die Untersuchungen standen Sauen der Rassen Deutsches Sattelschwein, Duroc, Mangalitza und Schwerfurter zur Verfügung, welche während der Weideperiode jeweils einen Wurf geboren und aufgezogen haben und wie folgt zu beschreiben sind:

- Schwerfurter Fleischrasse (SF), 20 Sauen: rahmig, wachstumsorientiert, gute Fleischfulle, mittlere Fruchtbarkeit und Streßresistenz;

- Deutsches Sattelschwein (DS), 20 Sauen: mittelgroße Tiere mit weißem Sattel, geringe Mast- und Schlachtleistungen, sehr gute Fruchtbarkeit, Streßresistenz und Fleischbeschaffenheit;

- Duroc (Du), 12 Sauen: rahmige, rotbraune Tiere, gute Mast- und Schlachtleistungen, geringe Fruchtbarkeit und Streßresistenz;

- Mangalitza (Ma), 5 Sauen: schwalbenbäuchiger Typ, Wollschwein ungarischer Herkunft, sehr geringe Mast- und Schlachtleistungen, geringe Fruchtbarkeit, sehr gute Streßresistenz.

Um die Wühlaktivität der einzelnen Versuchsgruppen zu bestimmen, erfolgte eine monatliche Erfassung. Die Wühlaktivität wurde mittels Transekt-Methode (HONE, 1980; PLACHTER, 1991; MÜHLENBERG, 1993) ermittelt. Dazu wurde ein QuadratmeterRahmen, der eine Einteilung in $10 \mathrm{~cm}^{2}$ besaß, verwendet. Dieser Quadratmeter-Rahmen ist in einem jeweiligen Abstand von $4 \mathrm{~m}$ auf je 2 Transekten je Untersuchungsfläche abgelegt worden. In jedem Quadratmeter wurde die prozentual umgewühlte Fläche geschätzt und die Wühltiefe gemessen. Aus diesen Werten errechnet sich die jeweils umgewühlte Fläche pro Monat und Versuchsgruppe sowie die Wühltiefe. Durch diese Transekt-Methode wurden auf den jeweiligen Flächen ca. $150 \mathrm{~m}^{2}$ untersucht.

Auf jeder Weidefläche sind von Beginn der Haltung der Schweine bis zum Vegetationsende jeden Monat je 5 zufällig verteilte, $1 \mathrm{~m}^{2}$ große Probeflächen, mit einer Schere in etwa 2-3 cm Höhe geerntet worden. Jede einzelne Probe wurde gewogen. Aus den jeweils 5 Proben je Versuchsfläche wurde nach der Wägung eine Mischprobe hergestellt. Aus dieser Mischprobe wurden 500g Frischmasse 24 Stunden bei $60^{\circ} \mathrm{C}$ getrocknet und anschließend zurückgewogen.

In den Monaten Juli bis November, während sich die Schweine auf der Weide aufhielten, wurden an je 2 Tagen pro Monat und Tiergruppe Verhaltensbeobachtungen durchgeführt. Es wurde zwischen den Verhaltensmerkmalen: Liegen, Grasen, Wühlen, Suchen, Stehen und Laufen unterschieden. In vorliegender Untersuchung wurde speziell 
auf die Merkmale Grasen, Wühlen und Liegen eingegangen. Die Verhaltensweisen sind im 10 min-Intervall (STOLBA und WOOD-GUSH, 1989) notiert. Beobachtet wurde von Sonnenaufgang bis Sonnenuntergang. Da sich die Tiere während der Nacht in ihren Hütten aufhielten, gingen diese Zeiten in das Merkmal Liegen ein. Damit beziehen sich die angegebenen Daten auf 24 Stunden.

Die Tiere sind bei Weideaustrieb und dann bis zum Abtrieb dreimal gewogen worden (Abb. 3).

Die Daten der Verhaltensbeobachtungen sind mit Hilfe des Statistical-Analysis-System (SAS, 1990) bearbeitet.

3. Ergebnisse und Diskussion

3.1 Wühlaktivität

3.1 .2 Wühlfläche

Bei Betrachtung der umgebrochenen Fläche (Abb. 1) fällt die geringe Wühlaktivität der Mangalitza auf, die etwa nur 1/3 der Fläche der Schwerfurter bzw. die Hälfte der Flächen des Deutschen Sattelschweines und der Duroc's brechen. In jedem Monat wiesen die Mangalitza die geringste, gebrochene Fläche auf. Die Schwerfurter brachen $28 \%$ der ihnen zur Verfügung stehenden Fläche und haben damit die höchste Wühlaktivität entwickelt. Das Deutsche Sattelschwein sowie die Duroc's brachen 19,0 bzw. 20,8\% der ihnen zur Verfügung stehenden Weidefläche um. Jedoch ist bei allen vier Schweinerassen eine Zunahme der Wühlaktivität von Juni zum November zu verzeichnen.

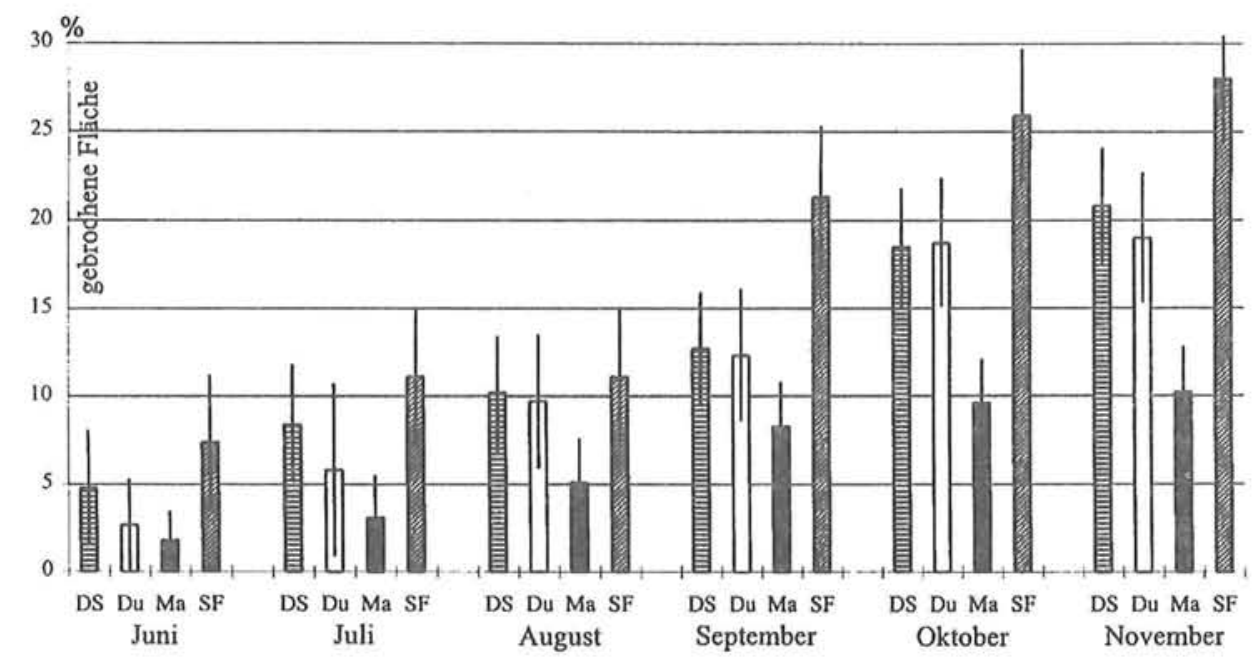

Abb.1: Quantitative Entwicklung der Wühlaktivität verschiedener Schweinerassen bei Freilandhaltung (Development of the rooting activity different breeds of pigs in outdoor keeping)

Das Wühlen der Schweine dient hauptsächlich dem Auffinden von Wurzeln, Knollen, Insekten (Larven und Puppen), Käfern, Regenwürmern, Kleinnagern und Reptilien 
JOST (1995). Durch die Suche der Tiere nach fressbaren Kleinlebewesen im Boden, bei gleichzeitig abnehmendem oberirdischen Futterangebot, nimmt die Wühlaktivität zum Herbst hin zu. Das bestätigen schon Untersuchungen von LUDWIG (1956), der im qualitativ niedrigeren Futterangebot die Hauptgründe für steigende Wühlaktivitäten sieht. Die Meinungen, sowohl über die Zunahme der Wühlaktivität wegen des abnehmenden oberirdischen Futterangebotes (PETERSEN, 1994) als auch über eine Stimulierung der Wühlaktivität in Zusammenhang mit lockerem Boden nach Regenfällen (SCHMID-VIELGUT u.a., 1991), können bestätigt werden.

SCHMĨD-VIELGUT u.a. (1991) regten Wildschein-Sauen zu intensiver Wühlaktivität nach Engerlingen an, indem ihnen als Zufutter nur pflanzliches Eiweiß (Rüben, Lupinen) angeboten wurde. In Mägen von Schwarzwild wurde vorwiegend pflanzliches Futter (Kräuter und Gräser) gefunden. Der Anteil pflanzlicher Nahrung betrug etwa $80-90 \%$ und der Anteil tierischer Nahrung entsprechend nur 10-20\%.

Mit fortschreitendem Vegetationsstadium nimmt der Rohfasergehalt des Weidefutters $\mathrm{zu}$, damit sinkt die Verwertbarkeit der anderen Inhaltsstoffe ab. Diese Tatsache ist mitverantwortlich dafür, daß die Tiere nach zusätzlicher tierischer und pflanzlicher Nahrung im Boden suchen, um ihren Futterbedarf zu decken.

Es ist festzustellen, daß es zur Zunahme der Wühlaktivität von Juni zum November kommt, welche darauf zurückzuführen ist, daß zum einen das ausreichende Angebot an oberirdischem Weidefutter abnimmt (Qualität) und zum anderen die Bodenbeschaffenheit im Herbst zum Wühlen besser geeignet ist, als beispielsweise im Sommer bei Trockenheit (Tab. 1). Feuchtes Herbstwetter begünstigt die Wühlaktivitätten (LUDWIG, 1956; SCHMID-VIELGUT u.a., 1991). Gleiche Beobachtungen über steigende Wühlaktivitäten ab August, September machten HONE und STONE (1989) in Nationalparks auf Hawaii und in Australien. SCHNEIDER und WALTER (1996) konnten mit zunehmendem Feuchtegehalt des Bodens ebenfalls eine Verschiebung des „Grasens“ zu Gunsten des „Wühlens“ feststellen.

Diese beiden Tatsachen scheinen die Hauptgründe für das verstärkte Einsetzen der Wühlaktivität zu sein, wobei sich zwischen den vier eingesetzten Rassen deutliche Unterschiede ergaben. Damit steht das Untersuchungsergebnis im Widerspruch zu einem Bericht von GRÜNENFELDER (1994), in dem er berichtet, daß MangalitzaSchweine als "Reservatpfleger" auf der Kiesinsel im Bodensee dazu eingesetzt werden, diese Insel vollständig umzugraben. Allerdings waren die Tiere während der Wintermonate auf der Insel und ein Rassenvergleich war nicht möglich, so daß diese Aussage nur bedingt zu werten ist.

\subsubsection{Wühltiefe}

Neben der umgebrochenen Fläche wurde die Wühltiefe festgestellt (Abb. 2). Die Tiere brechen die Grasnarbe in Tiefen von durchschnittlich 5 bis zu etwa $7 \mathrm{~cm}$. Hinsichtlich der Wühltiefe gibt es zwischen den verschiedenen Rassen keine großen Unterschiede. Sehr ausgeglichen scheinen im Jahresverlauf die Wühltiefen der Mangalitza zu sein, welche immer zwischen 4,4 cm (September) und maximal 5,5 cm (November) liegen und damit die signifikant geringsten Wühltiefen aufweisen. Die Duroc $(7,4 \mathrm{~cm})$ und 
die Schwerfurter $(7,5 \mathrm{~cm})$ haben im Juni und die Schwerfurter $(7,0 \mathrm{~cm})$ nochmals im August die tiefsten Wühlaktivitäten entwickelt. Tendenziell ist zum Herbst hin eine Abnahme der Wühltiefe festzustellen, wobei alle vier Rassen ähnliche Wühltiefen zeigen. Es wird deutlich, daß die Tiere unterhalb der Grasnarbe nach Nahrung im Boden suchen und deshalb die Weide umbrechen. Die mittleren, tieferen Wühlaktivitäten im Frühsommer und Sommer sind darauf zurückzuführen, daß die Tiere zu ihrer Abkühlung einzelne tiefe Mulden graben.

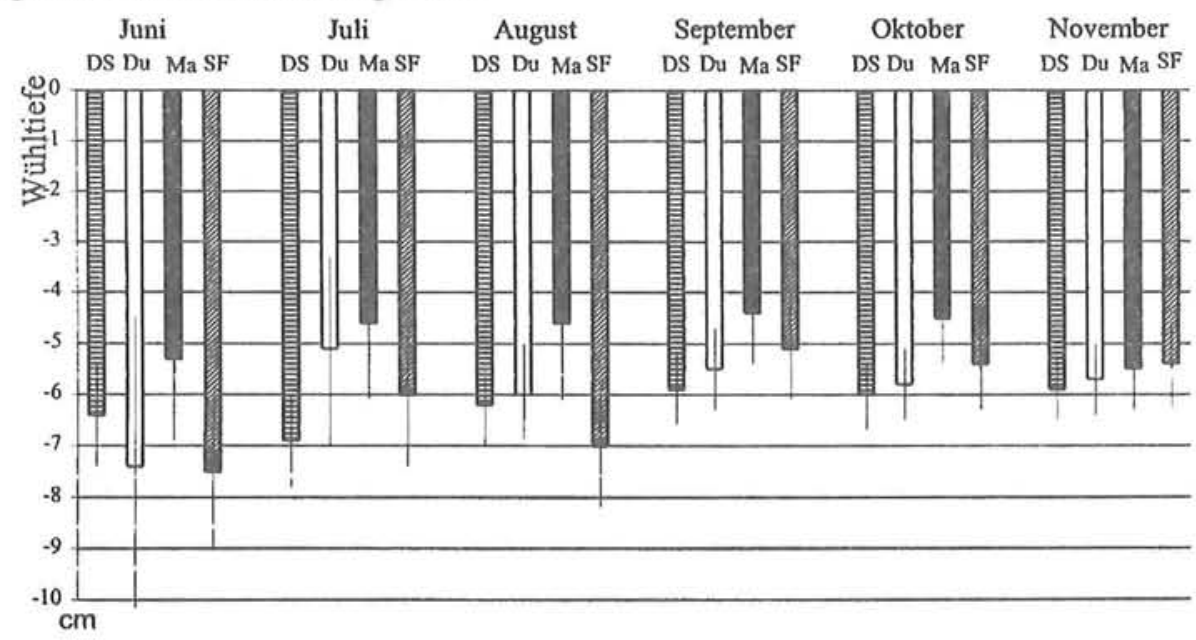

Abb. 2: Wühltiefen verschiedener Schweinerassen bei Freilandhaltung (Rooting depth of pigs in outdoor keeping)

BRIEDERMANN (1986) beschrieb das Oberflächenwulhlen mit Tiefen von $3-5 \mathrm{~cm}$ als sommerliche Hauptform der Nahrungsgewinnung. Er erwähnt diffuses Brechen, zur Gewinnung von tieferliegender Nahrung $(8-10 \mathrm{~cm})$ bis zu einer maximalen Ausdehnung von $40 \mathrm{~m}^{2}$, sowie stellenweises Brechen (besonders im Frühsommer) und Ausheben von Gruben zur Erreichung gewitterter Futterobjekte. Wildschweine im Great Smoky Mountains National Park (BRATTON, 1974) wühlten etwa 2 - $6 \mathrm{~cm}$, manchmal sogar bis $15 \mathrm{~cm}$ tief, um stärkehaltige Knollen, Zwiebeln oder Rhizome zu erreichen. Die Feststellungen können im wesentlichen bestätigt werden. Auch bei vorliegenden Untersuchungen setzte im Spätsommer das flächenweise Brechen der Grasnarbe ein, wobei Areale bis zu einer Größe von etwa 1 ha zusammenhängend gebrochen wurden. Hinsichtlich der Wühltiefe stimmen die Untersuchungen auch mit den beschriebenen Daten überein, wobei die mittlere Tiefe bei 5 bis $7 \mathrm{~cm}$ lag. Vereinzelt waren in geringerer Zahl tiefere Gruben (tiefer als $30 \mathrm{~cm}$ ) festzustellen, die neben der Nahrungssuche im Sommer als kühlende Liegeplätze genutzt wurden. Aktivitäten der Tiere im Verlauf der Freilandhaltung

Betrachtet man die Merkmale Grasen und Wühlen, so fällt auf, daß alle 4 Rassen im November signifikant weniger Grasen als im Juli, dafür aber signifikant länger Wühlen 
(vgl. Tab. 1). Im Juli grasen die DS etwa 9:00 Stunden und wühlen nur 12 Minuten pro Tag. Im November dagegen verbringen sie die doppelte Zeit (2:24 Stunden) mit Wühlen als mit Grasen (1:12 Stunden). Ähnliche Verhältnisse zwischen Grasen und Wühlen sind auch bei den anderen Rassen zu beobachten. Du und SF grasen im Juli 12:18 Stunden bzw. 8:00 Stunden und Wühlen im Mittel 12 bzw. 6 Minuten pro Tag. Im November verbringen die Du und SF 3:36 bzw. 5:48 Stunden mit Grasen und 5:24 Stunden bzw. 3:18 Stunden mit Wühlen. Die Ma zeigen in den Monaten Juli, August, September und November geringere Aktivitätszeiten als die anderen 3 Rassen. Im Oktober wiesen die Rassen DS und Du ebenfalls niedrigere Aktivitätszeiten als die SF auf. In den Monaten Juli bis November hatten die Ma stets die längsten Liegezeiten.

Tabelle 1

Weide- und Wühlverhalten verschiedener Schweinerassen im Verlauf der Weideperiode (Grazing and rooting activity different breeds of pigs in outdoor keeping)

\begin{tabular}{|c|c|c|c|c|c|c|c|c|c|c|c|}
\hline Rasse/Monat & Merkmal & $\begin{array}{c}\text { Juli } \\
\text { h:min }\end{array}$ & & $\begin{array}{l}\text { August } \\
\text { h:min }\end{array}$ & & $\begin{array}{c}\text { Sep. } \\
\text { h:min }\end{array}$ & & $\begin{array}{c}\text { Okt. } \\
\text { h:min }\end{array}$ & & $\begin{array}{l}\text { Nov. } \\
\text { h:min }\end{array}$ & \\
\hline \multirow[t]{2}{*}{ Dt. Sattelschw. } & Grasen & $9: 00$ & $\mathrm{~B}^{\circ}$ & $8: 06$ & & $8: 00$ & & $6: 18$ & & $1: 12$ & $\bar{B}$ \\
\hline & Wühlen & $0: 12$ & & $2: 54$ & A & $2: 48$ & A & $0: 24$ & C & $2: 24$ & B \\
\hline \multirow[t]{2}{*}{ Duroc } & Grasen & $12: 18$ & A & $12: 06$ & A & $11: 12$ & A & $6: 00$ & & $3: 36$ & \\
\hline & Wühlen & $0: 12$ & & $0: 12$ & B & $0: 36$ & B & $1: 30$ & B & $5: 24$ & A \\
\hline \multirow[t]{2}{*}{ Mangalitza } & Grasen & $6: 12$ & C & $5: 24$ & B & $5: 18$ & B & $4: 12$ & B & $3: 06$ & \\
\hline & Wühlen & $0: 06$ & & $0: 36$ & B & $1: 00$ & B & $1: 12$ & B & $1: 18$ & C \\
\hline \multirow[t]{2}{*}{ Schwerfurter } & Grasen & $8: 00$ & B & $8: 24$ & & $11: 36$ & A & $8: 06$ & A & $5: 48$ & A \\
\hline & Wühlen & $0: 06$ & & $0: 24$ & B & $0: 12$ & B & $2: 48$ & A & $3: 18$ & B \\
\hline
\end{tabular}

Tabelle 1 verdeutlicht, daß im Verlauf der Freilandhaltung von Juli bis November der Anteil der Tätigkeit Grasen bei allen vier Rassen abnimmt, wobei Wühlen ansteigt. Die Tiere verbringen im November deutlich mehr Zeit mit Wühlen als im Juli. Bei den Ma wurde im November die signifikant geringste Zeitwidmung für Wühlen (1:18 Stunden), verglichen mit den anderen Rassen, festgestellt.

Tabelle 2

Anteil „Liegen“ in \% der Gesamtaktivität während der Weideperiode („lying“ in per cent of the whole activity in outdoor keeping of pigs)

\begin{tabular}{ccccccccccc}
\hline Rasse/Monat & Juli & \multicolumn{3}{c}{ August } & Sep. & Okt. & & Nov. \\
\hline Dt. Sattelschw. & 46,7 & AB $^{*}$ & 40,4 & BC & 41,3 & B & 66,0 & A & 65,0 & A \\
Duroc & 45,2 & B & 40,6 & B & 42,0 & B & 63,7 & A & 56,4 & \\
Mangalitza & 65,4 & A & 56,0 & B & 54,2 & B & 60,1 & & 67,6 & A \\
Schwerfurter & 56,9 & A & 55,0 & & 40,0 & B & 47,1 & & 57,8 & A \\
\hline
\end{tabular}

Neben dem Grasen und Wühlen bildet die beobachtete Liegezeit den Hauptanteil aller Aktivitäten (Tab. 2). Während die Mangalitza die längsten Liegezeiten aufweisen, ist bei DS und DU ein Anstieg dieser Zeiten zum Ende der Weideperiode festzustellen. Bei den Schwerfurtern ist in den Monaten Juli, August und November eine Liegezeit von über $50 \%$ zu beobachten, während im Monat September sich diese Zeit vor allem zugunsten der Grasezeit und im Monat Oktober zugunsten der Wühlzeit reduziert. 
Tabelle 3 zeigt, daß den Tieren in den einzelnen Monaten Futter quantitativ in ausreichender Menge zur Verfügung stand. Im Verlauf der Vegetationsperiode nahm jedoch der Energiegehalt des Aufwuchses ab.

Tabelle 3

Aufwuchs und Energiegehalte des Weidefutters (Quantity and contents of energy in the fodder of the pasture)

\begin{tabular}{c|cc}
\hline Monat & $\begin{array}{c}\text { Aufwuchs } \\
\text { dt TS / ha }\end{array}$ & $\begin{array}{c}\text { Energiegehalte } \\
\mathrm{MJ} / \mathrm{kg} \text { TS }\end{array}$ \\
\hline Juni & 29,7 & 9,3 \\
Juli & 39,9 & 9,1 \\
August & 30,2 & 8,8 \\
September & 18,6 & 8,8 \\
Oktober & 22,9 & 8,7 \\
\hline
\end{tabular}

Futterprobenanalysen des Weidefutters ergaben mittlere Energiegehalte von 8,9 MJ ME / kg Trockensubstanz (TS). Für die verschiedenen Rassen ergeben sich damit folgende Energiemengen, die durch die Weidefutteraufnahme gedeckt (Tab. 4) sowie die entsprechenden Futtermengen in Originalsubstanz (OS), die durch die Tiere aufgenommen werden.

Tabelle 4

Durchschnittliche Lebendmasse, Erhaltungsbedarf sowie notwendige Weidefutteraufnahme vier verschiedener Schweinerassen (Life weight in average, reqiurement of maintenance and necessary reception of fodder different breeds of pigs)

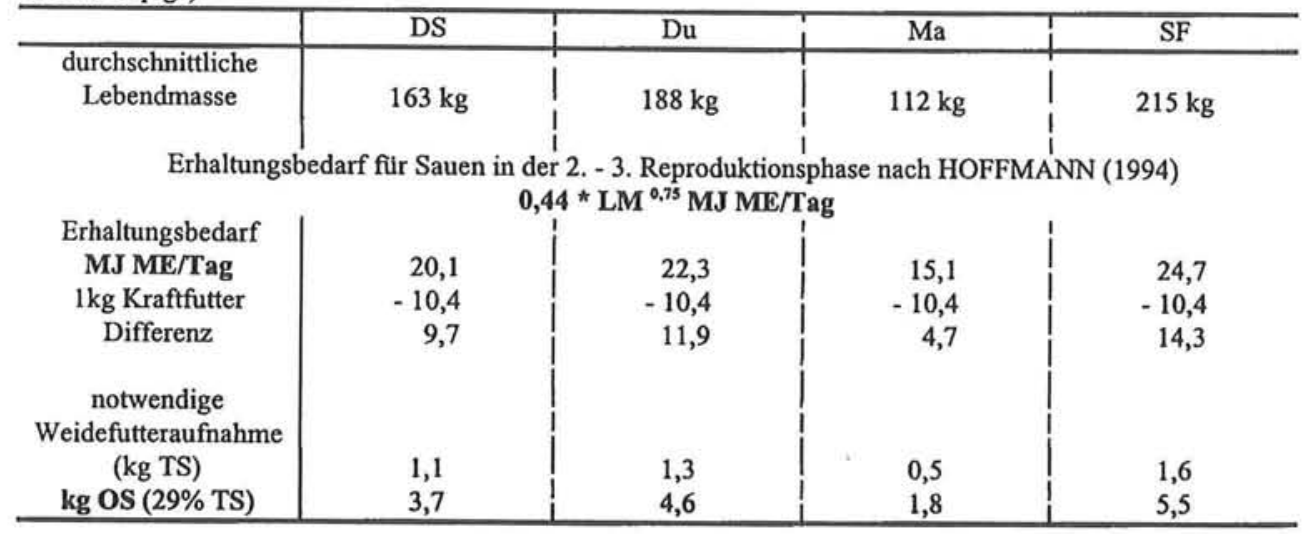

Die rechnerisch ermittelten notwendigen Trockensubstanzaufnahmen stimmen mit Angaben von BEYER u.a. (1995) über Trockensubstanzaufnahmen bei güsten und graviden Sauen gut überein. JANDA (1958) gibt Nahrungsmengen in Mägen von Wildschweinen mit durchschnittlich 2,5 kg an, wobei FORSTNER (1967) diese Zahlen bezweifelt und es nach seinen Untersuchungen nur etwa $1 \mathrm{~kg}$ sein sollen. HECK und RASCHKE (1980) geben ein Mageninhaltsgewicht eines $77 \mathrm{~kg}$ schweren Überläufers von 1,5 kg an. Für den Gesamttagesbedarf einer Sau sprechen sie von einem Gewicht von etwa $4 \mathrm{~kg}$. Nach BOGNER und GRAUVOGEL (1984) können Altsauen bis zu 18 kg Weidefutter aufnehmen. PORZIG und SAMBRAUS (1991) sprechen bei der 
Weidefutteraufnahme von Sauen über etwa $10 \%$ ihrer Lebendmasse.

Die tägliche Ration während der Trächtigkeit soll $300 \mathrm{~g}$ Rohprotein und 200-240 g verdauliches Rohprotein enthalten. Durch das Futterangebot und die tägliche Zufütterung erhielten die Tiere ca. $485 \mathrm{~g}$ Rohprotein bzw. $165 \mathrm{~g}$ verdauliches Rohprotein. Die Versorgung mit Rohprotein lag damit unter der empfohlenen Menge. JOST (1995) bemerkt, daß bei Zuchtsauen die Verwertung von rohfaserreichen Futtermitteln um 10 - $20 \%$ höher liegen soll, als in den Nährwerttabellen angegeben wird. Die bessere Verwertung resultiert aus einer intensiven bakteriellen Fermentation im Dickdarm (JOST, 1985).

Mit abnehmender Futterqualität im Herbst müssen die Schwerfurter nach Futter im Boden suchen. Aus Gründen des höheren Erhaltungsbedarfes, einer höheren Stoffwechselrate und der hauptsächlichen Futtersuche im Boden, brechen die Schwerfurter den größten Anteil der Fläche. Den Deutschen Sattelschweinen und den Duroc's als „anspruchslosere Weideschweine“ genügt das Futterangebot der Weide und im Boden, um ihr Gewicht zu halten.

Die durchschnittlichen Lebendgewichte der Tiere (Abb. 3) lassen einen deutlichen Abfall der Gewichte der SF zum Vegetationsende erkennen, während die Mangalitza nach dem 5. Oktober noch zunahmen.

Der Grund für die größeren Gewichtsverluste der auf eine große Fleischfülle gezüchteten Schwerfurter liegt in einer ungenügenden Energieversorgung der Schweine wegen der abnehmenden Weidefutterqualität begründet. Dieser Typunterschied erklärt auch die relative Gewichtskonstanz der Mangalitza während der Versuchsdauer.

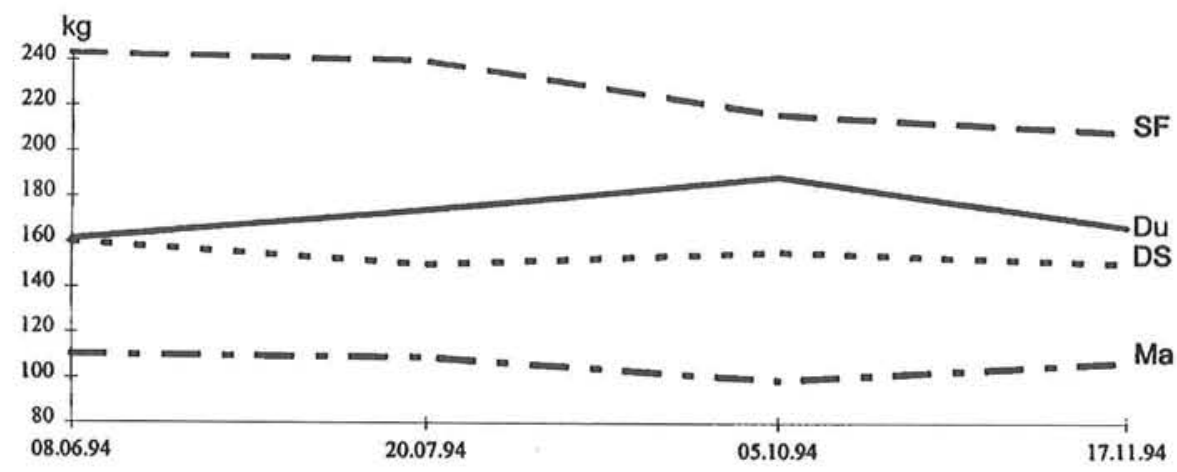

Abb. 3: Gewichtsentwicklung vier verschiedener Schweinerassen bei Freilandhaltung (Development of life weight different breeds of pigs in outdoor keeping)

Der Hauptteil der aufgenommenen Energie wird über Wärme wieder abgegeben (BEYER u.a., 1994). Möglicherweise reicht die angebotene Energie des Weide- und Zufutters im Herbst, besonders bei den SF (Abb. 3), nicht mehr aus, um den erhöhten Anteil für die Wärmeproduktion im Herbst auszugleichen.

Anhand der Tiergewichte kann man feststellen, daß die Deutschen Sattelschweine, die Duroc und auch die Mangalitza hinsichtlich ihrer Lebendmasse-Entwicklung extensive 
Freilandhaltungsbedingungen relativ gut bewältigen, während der Weideperiode keine extremen Gewichtsverluste erfahren und trotz Wurfleistungen bei Weideabtrieb ihre Auftreibsgewichte erreichten. Im Rassenvergleich ergab sich, abgeleitet aus der Gewichtsentwicklung und der Wühlaktivität eine gleich gute Eignung für die extensive Weidehaltung.

Die Schwerfurter, als wüchsige Intensivrasse, konnten trotz hoher Wühlaktivität ihren Energiebedarf, im Gegensatz zu den Mangalitza, nur ungenügend decken. Desweiteren ist zu bemerken, daß während der gesamten Versuchsdauer zwei Sauen der Rasse Schwerfurter während einer langen Hitzeperiode verendeten. Der geringere Energiebedarf der Mangalitza führt offensichtlich auch zur beobachteten, geringeren Wühlaktivität. Bedingt durch eine geringere Lebendmasse, haben die Mangalitza einen wesentlich geringeren Erhaltungsbedarf (15,1 MJ ME / Tag) zu decken als die Schwerfurter (24,7 MJ ME / Tag).

\section{4}

Wurfleistungen

In Tabelle 5 sind die Abferkelergebnisse sowie die Ferkelverluste während der Freilandhaltung dargestellt. Deutlich erkennbar ist der relativ hohe Verlust an Ferkeln unter diesen Freilandhaltungsbedingungen.

Tabelle 5

Abferkelergebnisse verschiedener Schweinerassen unter Freilandhaltungsbedingungen (Results of farrowing different breeds of pigs in outdoor keeping)

\begin{tabular}{lcccc}
\hline & Schwerfurter & $\begin{array}{c}\text { Deutsches } \\
\text { Sattelschwein }\end{array}$ & Duroc & Mangalitza \\
\hline Anzahl Würfe & 20 & 20 & 12 & 5 \\
Geburtsgewicht (kg) & 1,4 & 1,5 & 1,3 & 1,2 \\
Anzahl geb. Ferkel & 10,6 & 8,7 & 8,8 & 5,6 \\
Absetzgew. (kg) & 10,3 & 9,9 & 8,3 & 10,6 \\
Anzahl abges. Ferkel & 7,6 & 6,2 & 6,3 & 4,4 \\
Säugezeit (Tage) & 38,9 & 40,9 & 40,3 & 77,6 \\
tgl. Zunahme (g) & 215,3 & 203,3 & 172,5 & 122,8 \\
Verluste absolut & 3,0 & 3,0 & 3,8 & 1,2 \\
Verluste relativ (\%) & 25,5 & 32,1 & 42,2 & 23,7 \\
\hline
\end{tabular}

Die Ferkelverluste schwanken zwischen 1,2 Ferkeln bei den Mangalitza und 3,8 Ferkeln bei den Duroc, wobei zu berücksichtigen ist, daß sich die Geburtswurfgrößen bei den vier Rassen erheblich unterschieden. Der Hauptgrund für die Ferkelverluste war das Erdrücken.

Im Vergleich zwischen Deutschem Sattelschwein und Duroc erreicht das Deutsche Sattelschwein bessere Aufzuchtergebnisse. Die Schwerfurter brachten die höchsten Zahlen an geborenen und aufgezogenen Ferkeln.

Damit kann festgestellt werden, daß unter den gegebenen Untersuchungsbedingungen höhere Ferkelverluste auftraten, als bei SPITSCHAK (1997a,b), der bei Freilandhaltung nur Verluste von im Mittel 16,6\% bei einer Säugezeit von 22,9 Tagen feststellte. MAJOR (1986) hatte sogar nur 10,2\% Verluste bei einer Säugezeit von 28 Tagen, wobei in beiden Arbeiten bessere Standortbedingungen vorlagen und die Ferkelproduk- 
tion Ziel der Sauenhaltung war.

Die hier vorgestellten Ergebnisse zeigen, daß diese extensive Form der Freilandhaltung von Sauen mit Ferkelerzeugung bei geringstem Aufwand, eine Nebennutzung derartiger Flußauenstandorte ermöglicht. Obwohl die sechsmonatige Freilandhaltung von den Tieren aller Rassen gesundheitlich gut vertragen wurde, erwiesen sich die extensiven Rassen als besser geeignet für die Weidehaltung jedoch weniger geeignet für die Ferkelproduktion, wobei die Schwerfurter bei Gewichtsverlusten während der Weidedauer relativ gute Ferkelaufzuchtergebnisse erreichten.

\section{Literatur}

BEYER, M.; JENTSCH, W.; HOFFMANN, L.; SCHIEMANN, R.:

Untersuchungen zum Energie- und Stickstoffumsatz von graviden und laktierenden Sauen sowie von Saugferkeln. 5. Mitt.: Energie- und Stickstoffumsatz von graviden Sauen. Archives of Animal Nutrition 46 (1994), 173-205

BEYER, M.; JENTSCH, W.; HOFFMANN, L.; SCHIEMANN, R.:

Untersuchungen zum Energie- und Stickstoffumsatz von graviden und laktierenden Sauen sowie von Saugferkeln. 6. Mitt.: Vergleichende Energie- und Stickstoffumsatzmessungen an güsten und graviden Sauen. Archives of Animal Nutrition 47 (1995), 187-217

BOGNER, H.; GRAUVOGL, A.:

Verhalten landwirtschaftlicher Nutztiere. Verlag Eugen Ulmer, Stuttgart, 1984

BRATTON, S.P.:

The effect of the europeanwild boar (Sus scrofa) on the high-elevation vernal flora in Great Smoky BRIEDERMANN, L.:

Schwarzwild. Deutscher Landwirtschaftsverlag, Berlin, 2.Auflage, 1986

DURST, L.; WILLEKE, H.:

Freilandhaltung von Zuchtsauen. KTBL-Schriften, Arbeitspapier 204, Darmstadt, 1994

FORSTNER, L.:

GLODEK, P.: Wildäsung und Wildfütterung. Der Anblick 22 (1967), 283-284

Schweinezucht. Tierzuchtbücherei, Verlag Eugen Ulmer, Stuttgart, 1992

GRUNENFELDER, H.P.:

Erhaltung der Wollschweine, ein internationales Bemühen. Unser Land 4 (1994), 30-31

HECK, L.; RASCHKE, G.:

HOFMANN, F.:

Die Wildsauen, Naturgeschichte-Ökologie-Hege und Jagd. Verlag Paul Parey, Hamburg, Berlin, 1980

Öber den Stand der Freilufthaltung von Schweinen und Rindern in Thüringen. Die Deutsche HONE, J.: Landwirtschaft 10 (1952), 547 - 549

Effect of feral pig rooting on introduced and nativ pasture in north-eastern New South Wales. Journal of the Australian Institute of Agricultural Science 46 (1980), 130-132

HONE, J.; STONE, C.:

A comparison and evaluation of feral pig management in two National Parks. Wildlife Society Bulletin JANDA, M.: 17 (1989), 419-425

Die Nahrung des Schwarzwildes, Sus scrofa L., im Mittelgebirgsgebiet von Stiavnica. SäugetierkundJOST, $\mathrm{M}$ : liche Mitteilungen 6 (1958), 67-74 JOST, M.

Grundfutter an Sauen - aber wie? Schweinezucht und Schweinemast 33 (1985), 143-146

Weidehaltung von Schweinen. Landw. Schweiz 5 (1992), 521 
JOST, M.:

Freilandschweine richtig füttern. Agrarforschung 2 (1995), 68-69

KLAPP, E., OPITZ VON BOBERFELD, W.:

Kräuterbestimmungsschlüssel für die häufigsten Grünland- und Rasengräser. Verlag Paul Parey, Berlin, Hamburg, 2. Auflage, 1988

LUDWIG, A.:

MAJOR, R.:

Haltung und Fütterung von Schweinen auf Dauerweiden. Univ. Stuttgart, Hohenheim, Diss., 1956

Ferkelerzeugung in Freilandhaltung. Schweinezucht und Schweinemast 34 (1986), 288-292

MEISEL, K.; HÜBSCHMANN, v. A.: MEISEL, $\mathrm{K}$ :

Grundzige der Vegetationsentwicklung auf Brachflächen. Natur und Landschaft 48 (1973), 70-74

Über Umfang, räumliche Verteilung und Vegetationsentwicklung von Brachflächen in der BRD. Jahrbuch für Naturschutz und Landschaftspflege 22, Bonn - Bad Godesberg, 9-27, 1973

MICKLICH, D.:

Die Wirkung der Weidehaltung verschiedener Schweinerassen auf die Sukzession von MÜHLENBERG, M:

Freilandökologie. Quelle und Meyer, UTB-Taschebücher, 3. Auflage, 1993

PETERSEN, V.:

The development of feeding and investigatory behaviour in free-ranging domestic pigs during their first 18 weeks of life. Applied Animal Behaviour Science 42 (1994), 87-98

PLACHTER, H:

Naturschutz. UTB für Wissenschaft, Gustav Fischer Verlag, Stuttgart, Jena, 1991

PORZIG, E.; SAMBRAUS, H.H.:

Nahrungsaufnahmeverhalten landwirtschaftlicher Nutztiere. Deutscher Landwirtschaftsverlag, Berlin, 1991

REICHEL, D.:

Zur ökologischen Beurteilung von Brachflächen. Berichte der Akademie für Naturschutz und RUNGE, F.: Landschaftspflege1, 36-42, 1977

21-, 10- und 8-jährige Dauerquadratuntersuchungen in aufgelassenen Grünländereien. In: SCHREIBER, K.-F.: Sukzession auf Grünlandbrachen, Münstersche Geographische Arbeiten, Heft 20, Ferdinand Schöningh Verlag, Paderborn, 45-50, 1985

SAMBRAUS, H.H.:

Sauenhaltung tier- und leistungsgerecht. In: SAMBRAUS, H.H., BOEHNCKE, E.: Ökologische Tierhaltung. Theoretische und praktische Grundlagen für die biologische Landwirtschaft. Verlag C.F. Müller, Karlsnuhe, 1986

SAS INSTITUTE INC.:

SAS User's Guide. Vol 2, LM-VARCOMP, Version 6, 4. Edition (1990)

SCHLEGEL, W.; RITTER, E.:

Moderne Ferkelaufzucht. Deutscher Bauernverlag, Berlin, 1959

SCHMID-VIELGUT, B.; DOPF, M.; BOGENSCHÜTZ, H.:

Einfluß von gegattertem Schwarzwild auf die Populationsdichte des Waldmaikäfers. Allgemeine Forstzeitschrift für Waldwirtschaft und Umweltvorsorge 14 (1991), 719-721

SCHMIDT, D.; SCHLICHTING, M.C.; LADEWIG, J.; STEINHARDT, T.M.:

Ethologische und verhaltensphysiologische Forschung für tiergerechte Nutztierhaltung. Arch. Tierz., Dummerstorf 38 (1995), 7-19

SCHNEIDER, P.; WALTER, J.:

Ethologische Untersuchungen von Mastschweinen auf Weiden. Arch. Tierz., Dummerstorf 39 (1996), 299-307

SPITSCHAK, K.:

Fruchtbarkeits- und Aufzuchtleistungen von Sauen mit Ferkeln in der Freilandhaltung. Arch. Tierz., Dummerstorf 40 (1997a), 123-134 
SPITSCHAK, K.; FRANKE, W.:

Freilandhaltung von Sauen und Ferkeln - mehr als nur eine Alternative. Arch. Tierz., Dummerstorf 40 (1997b) Sonderheft, 35-40

STOLBA, A.; WOOD-GUSH, D.G.M.:

The behaviour of pigs in a semi-natural environment. Animal Prod., Edinburgh 48 (1989), 419-425

Eingegangen: 13.02 .1998

Akzeptiert: 11.02 .1999

\begin{abstract}
Anschriften der Verfasser
Dr. DELIA MICKLICH

Eickhöfer Weg 5

D-18209 Bad Doberan
\end{abstract}

Dr. habil. HEIDE-DÖRTE MATTHES

Forschungsinstitut für die Biologie landwirtschaftlicher Nutztiere (FBN)

Wilhelm-Stahl-Allee 2

D-18196 Dummerstorf 


\section{Buchbesprechung}

Mengen- und Spurenelemente - 18. Arbeitstagung 1998, Friedrich-Schiller-Universität, Jena

\section{MANFRED ANKE und Herausgebergruppe}

Bericht der 18. Arbeitstagung vom 4, und 5. Dezember 1998 in Jena. Organisation: Friedrich-Schiller-Universität, Jena sowie weitere Universitäten und Einrichtungen, 1055 Seiten, zahlreiche Tabellen und Abbildungen, Verlag Harald Schubert, Leipzig 1998, ISBN 3-929528-45-X

Das Interesse von Wissenschaft und Praxis an der biologischen, medizinischen, toxikologischen, nutritiven, land- und forstwirtschaftlichen Bedeutung der Mengen- und Spurenelemente ist weiterhin ansteigend. Das wird u.a. durch den Umfang des Tagungsberichtes deutlich. Enthielt der 17. Band noch 121 Vortrags- und Posterberichte, hatte der Veranstalter zur 18. Tagung 145 Manuskripte angenommen, von denen bis auf 9 alle in den vorliegenden Tagungsband Eingang fanden. Das internationale Interesse an dieser Problematik wird auch durch die Tatsache belegt, daß die Autoren 24 Länder repräsentieren, die, außer aus Australien, von allen Erdteilen kamen. Im Wissen um die Zusammenhänge Umwelt-Boden-Pflanze-Tier-Mensch beschäftigt diese Thematik, neben ihrer fachwissenschaftlichen Bedeutung, eine immer breitere Öffentlichkeit unter dem Aspekt Nahrung und Gesundheit von Mensch und Tier. Diesem Umstand wird auch thematisch in der ubergroßen Zahl der Bejträge Rechnung getragen.

Das fachwissenschaftliche Spektrum der Beiträge ist von der Sache her begrïndet sehr breit. Neben analytischen und methodischen Arbeiten sind vor allem solche Beiträge enthalten, die sich mit den Auswirkungen von Mangel- oder Überversorgung der einzelnen Mengen- bzw. Spurenelementen befassen. Viele Beiträge beschäftigen sich mit Fragen dieser Elemente in Nahrungsmitteln, Futtermitteln oder mit ihrer Bedeutung in der Human- oder Veterinärmedizin. Aus der Fülle der Komplexe seien solche genannt wie Zusammenhänge zwischen Mengenund Spurenelementen in der Nahrungskette und Industriestandorten, Schwermetalltransfer in der Nahrungskette, Versorgungsstatus mit Spurenelementen, Belastungen durch dentale Werkstoffe, Wechselwirkung zwischen Nahrungsbestandteilen und Nahrungsstoffen, Metalle und Immunsystem usw. Die Vielzahl der Beiträge bietet nicht nur umfangreiche Informationen und Anregungen, sondern ermöglicht durch die angeführten Autorenadressen Kontakte für weiterführende Gespräche zwischen Fachkollegen und Forschungsgruppen im Inund Ausland.

Wie bereits erwähnt, brachte die umfangreiche internationale Beteiligung auch eine Veränderung in der Vortragssprache mit sich. So sind inzwischen mehr als die Hälfte der Arbeiten im Tagungsbericht in englischer Sprache verfaßt worden.

Ein nach Mengen- und Spurenelementen geordnetes Sachverzeichnis erleichtert die Arbeit mit diesem Tagungsbericht. Das Autorenverzeichnis beschließt dieses wiederum außerordentlich informative, interessante, und einer überaus aktuellen Thematik gewidmete Buch, dem eine große Leserschar und Verbreitung zu wünschen ist. 